\title{
Mucopolysaccharidosis type 2
}

INSERM

\section{Source}

INSERM. (1999). Orphanet: an online rare disease and orphan drug data base.

Mucopolysaccharidosis type 2. ORPHA:580

Mucopolysaccharidosis type 2 (MPS2) is a lysosomal storage disease leading to a

massive accumulation of glycosaminoglycans and a wide variety of symptoms including distinctive coarse facial features, short stature, cardio-respiratory involvement and skeletal abnormalities. It manifests as a continuum varying from a severe to an attenuated form without neuronal involvement. 